\title{
Grounding of Human Observations as Uncertain Knowledge
}

\author{
Kamil Szymański and Grzegorz Dobrowolski \\ AGH University of Science and Technology, Cracow, Poland \\ camel_sz@go2.pl, grzela@agh.edu.pl
}

\begin{abstract}
The article presents some methods of uncertain, humanoriginated knowledge grounding, accomplished with the use of ontologies with uncertainities and several ontology ABox joining techniques. Several people with uncertain knowledge about some domain write down their domain knowledge in a form of simple text in english. The goal is to obtain uncertain ontology ABoxes, representing their knowledge, and to join them, resulting in total, grounded, uncertain domain knowledge. The joining process takes care of different facts description details, possible facts conflicts as well as varied facts certainity degrees and people credibility.
\end{abstract}

Keywords: uncertain knowledge, uncertain knowledge grounding, ontology ABoxes joining, human-originated knowledge.

\section{Introduction}

The paper is a proposition of solving the task of uncertain, human-originated knowledge grounding. Several people with uncertain knowledge about some domain write down uncertain domain facts in a form of simple text in english. The goal is to provide one, total uncertain domain knowledge, based on mentioned information pieces, that can vary in described facts, their certainities and description details. It is also necessary to take into consideration different people credibilities and possible facts conflicts. The solution presented in the paper is based on automated building of several domain ontologies [4 with uncertainities from the text (ABoxes only, with constant TBox supplied externally). Subsequently, ABoxes of the ontologies are joined 212 in a way that reflects total knowledge the most adequately. The resulting ontology contains evaluated certainity degrees of every sentence as well, depending on subjective certainity degrees of knowledge contributors and their credibilities.

\section{Solution Concept}

The whole process of knowledge grounding presented in this paper is shown in Fig. 1. The abstract TBox, called also the base TBox, is a kind of upper ontology 9] for all possible domain TBoxes. It contains several basic concepts:

M. Bubak et al. (Eds.): ICCS 2008, Part III, LNCS 5103, pp. 555-563, 2008.

(C) Springer-Verlag Berlin Heidelberg 2008 


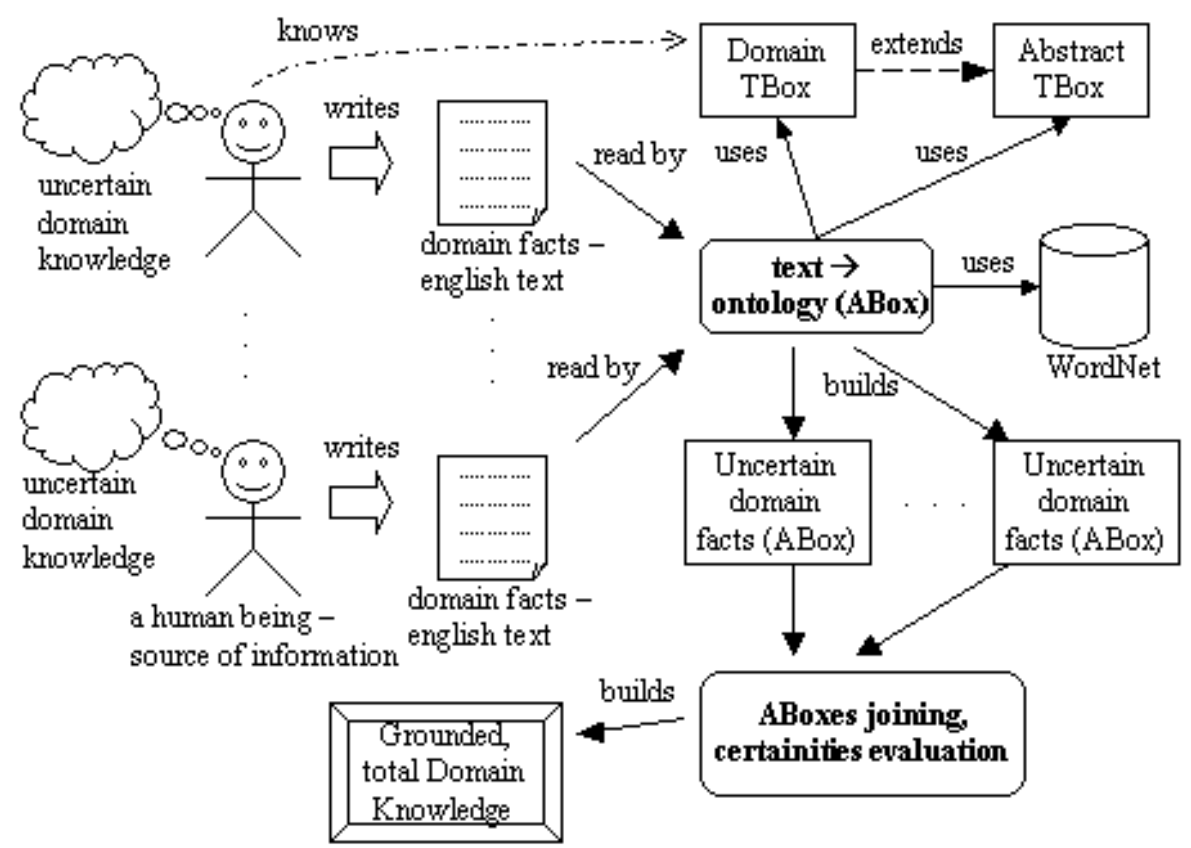

Fig. 1. Solution concept

Entity - Existence, for representing classes of objects, Relation - Relation, for representing relations between two entities, a subject and an object, and Property - Property, which represents entity attributes. Among relations, there are actions and states. In the abstract TBox it is also modelled that each action can have many following actions. It is important for events chronology. Among properties, there are numerical properties, NumericalProperty, and enumerative properties, EnumProperty, the latter having a finite set of values.

The domain TBox contains concrete, domain terminology. All its concepts should extend a proper base concept from the abstract TBox. Domain concepts, like $a$ car or a person should be subclasses of Existence, while concrete action hitting and state has color should inherit abstract action and state relations respectively. Speed, Height or Age could serve as domain examples of NumericalProperty.

There are several human beings, sources of information, who have knowledge about some domain. The knowledge is uncertain as well as different people have varied credibilities. To model those uncertainities in ontologies [13]14, which were chosen for knowledge representation, several certainity factors are introduced, with values within $\langle-1 . .1\rangle$, where 1 means total certainity and $-1-$ total contradiction. There are three kinds of relation certainities:

- relationCertainity - performing action certainity

- relationSubjectCertainity - the action subject certainity

- relationObjectCertainity - the action object certainity 
A similar certainity degree for entity attributes can be defined - let it be called propertyCertainity. All degrees mentioned above are related to subjective opinions of a source of information and can be assigned to each sentence. Additionally each person has yet another certainity degree assigned that reflects his credibility. Each certainity value present in a sentence must be multiplied by the credibility factor of the information source.

It is assumed that people know domain terminology — the domain TBox. They write down their domain knowledge in a form of a piece of text in english. This is where knowledge processing, described in the article, begins. Firstly, all pieces of text are parsed in order to extract domain ABoxes - known domain facts.

Then, previously obtained ABoxes are joined using several techniques, like identifier-based joining, pattern-based joining, action sequences joining (used in domains describing events with many actions, where chronology is an important factor) or closure-based joining. For output ontology, although grounded, after using mentioned methods, is still uncertain, proper certainity evaluation formulas needed to be suggested as well. All phases of the process and used methods are more precisely described in the following sections.

The implemented solution with text parsing is temporary as it has a few disadvantages. Firstly, a person writing the text, must know what sentences are permissible, in what order they should be written for the resulting ontology to be correct and logical and what domain aspects are served. Those requirements are hard to fulfill in real conditions. Secondly, there is no way to assure that all needed information were written - the writer could still forget to mention some facts he knows about. Those flaws will greatly be limitted when a domain-aware agent is introduced. The agent will lead the dialog with a person, traversing the domain ontology and available terminology, thus restricting the role in the whole process of a human being to giving the answers to concrete, precise questions. This intelligent agent will have the ability of asking proper questions, because of its converstation history and context awareness. The abilities of the agent are thought to be similar to those described in [6].

\section{Domain ABoxes Building}

At first, the text is formatted and all unnecessary phrases and prepositions are deleted for every sentence in the text to be identified. Then, semantic analysis of each sentence takes place. It is done using WordNet project [1]. A list of the most probable parts of speach, like a noun or a verb, is created for every word in each sentence. The building module, basing on a list of available sentence schemes in the form of

〈subject, relation, object, sentence certainity phrase〉,

builds the domain ontology connected with the concrete person, contributing to the knowledge. Several associate lists, which need to be provided externally, are used, such as 
$\langle$ certainity phrase, certainity value $\rangle$, eg. 〈'maybe', 0.4 $\rangle$, 〈'certainly', 1.0, or base speach forms to a corresponding class in ontology mapping, like

$$
\begin{gathered}
\text { 〈'getting closer', class:Approaching }\rangle, \\
\langle(\text { 'Toyota','Avensis'), class:ToyotaAvensisCar }\rangle \text {. }
\end{gathered}
$$

The 'it' preposition is also served. This way, the subject of the current sentence does not need to be repeated in the following one as long as it stays the same. Instances are identified and created in the knowledge base as well.

\section{Domain ABoxes Joining}

Having all domain ABoxes, forming knowledge from several human beings, the joining process 38 can be started to obtain aggregate, complete knowledge. Different credibilities of sources of information or points of view and possible missing facts must be taken into account while performing ontology ABoxes joining.

\subsection{Identifier-Based Joining}

The idea of performing joining based on identifiers has its origin in assumption that in real world there are certain entity kinds for which an identification system was created, like unique car licence plates. Two objects with the same identifiers are found to be synonims. The big advantage of the method is high matching accuracy, but from the other hand the amount of such entity types is rather small.

\subsection{Pattern-Based Joining}

An instance can be described by a set of numerical properties, like speed or height, and discrete properties with finite number of possible values, such as colours. If two individuals represent the same class and their corresponing properties' values are similar, there is a chance for these two instances to be synonims. A pattern is a set of elements of the following two types:

- A discrete role - its values must be equal in comaparing instances, like sex,

- A numerical role with fault margin - certain differences in values are permitted

All patern conditions have to be met to make two instances synonims. This method works the best if the domain is not too broad and the number of actors is not large.

\subsection{Action Sequences Joining}

A specification of events chronology in events-oriented domains is one of the most important things to be taken care of. Matching action types and succession is 
an important factor in synonims searching between corresponding actions and their actors. The analysis of possible successive actions for every action in domain ABoxes results in directed graph of action succession creation. Then, the action succession graph need to be linearized, using the breadth-first searching algorithm. For example, after linearization of the graph from the Fig. 2 (left side), the following action sequences will be created: (A, B, E, F), (A, C, E, F), (A, D, $\mathrm{F})$. After all graphs linearization is complete, a comparison between each pair of
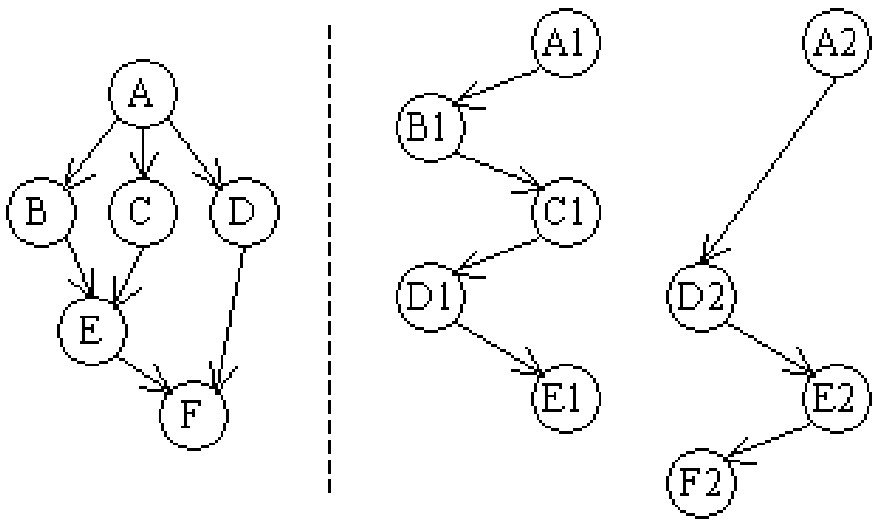

Fig. 2. An example action graph (left), Action sequences (right)

sequences from different ABoxes is performed. The comparison results in correspondence sequence detection, which consists of potentially synonimical actions. For an action pair to be accepted to the correspondence sequence, both actions have to be of the same kind. Additionally, the actions' subjects or objects have to be of the same class or classes near each other in class hierarchy (if the classes represent detailed concepts and only for certain, configurable cases).

Compared action sequences can, and probably will be only similar, not equal, because different descriptions of a complex event may vary in details. That is why not all actions from the sequence have to form a correspondence sequence - only matching ones. For example, in case of action sequences from Fig. 2 comparison, a correspondence sequence ((A1, A2), (D1, D2), (E1, E2)) will be created. From all correspondence sequences found for a certain pair of ontologies, the longest sequence is chosen. All its paired actions are considered synonims if the sequence length is more than three.

\subsection{Closure-Based Joining}

Ontologies can be treated like a connection graph with individuals or actions as vertices and hasSubject or hasObject relations as edges. The main idea behind closure-based joining is an assumption that if, for example, two individuals (verticies in the connection graph) are synonims, their adjoining vertices (for 
example two other individuals) could be synonims as well unless they are not similar or have different types.

Two kinds of closures are defined:

- action closure - if two actions have the same type and their subjects and objects are synonims, the actions are synonims as well,

- subject (object) closure (entity closure) — if two actions are synonims and their objects (subjects) are synonims, their subjects (objects) are synonims as well.

These closures are applied multiple times so as synonims discovery propagates through the connection graph.

\subsection{Certainities Evaluation}

If two facts do not collide with each other, there is not any problem with them both are taken into the joined ABox as they were in domain ABoxes. Undoubtly there will be some colliding facts too, and not colliding facts, but with different certainity factors. In the first case, a fact with the highest certainity value must be taken, in the latter - although there is no argue concerning the fact itself, its final certainity degree needs to be calculated.

A fact with different certainity values. Let the fact in question has certainity values $p_{1}, p_{2}, \ldots, p_{n}$, coming from information sources $1 . . n$. These values already include credibilities $w_{1} . . w_{n}$ of sources $\left(p_{i}=w_{i} * q_{i}\right.$, where $q_{i}$ is a subjective opinion of the $i^{\text {th }}$ source about the fact). The final certainity $p$ is the mean of $p_{i}$ values.

Colliding facts. In this case one of the facts need to be taken and its final certainity value must be evaluated. There are two possibilities, depending on property kind:

- discrete properties - a property value with the highest certainity degree need to be chosen. Let property values $a_{i},(i=1 . . n)$ have among $n$ people certainities $p_{i, 1} . . p_{i, k(i)},(1 \leq k(i) \leq n)$, and these certainities include sources credibilities (the same as previously). Property value $a_{x}$ with the highest mean certainity value $p_{x}=\sum_{j=1 . . k(x)} p_{x, j} / k(x)$ is taken into the joined ABox. The value $p_{x}$ is an output certainity value of the fact as well.

- numerical properties with fault margin - in this case two values are considered not equal if their difference is greater than the fault margin. Otherwise, the means of the values and their certainities $\left(p_{i}\right)$ are calculated and treated like a common value. The rest of the procedure is analogous to the previous case, barring the difference that now the calculations can be performed many times for different mean combinations (eg. when $a$ and $b$ are within the fault margin, $b$ and $c$, too, but $a$ and $c$ not any more, both the mean of $a$ and $b$ and the mean of $b$ and $c$ must be taken into account, in different iterations). When the means are evaluated, the number of elements forming the means have to be remembered, because those values must have proportionally higher wage in following evaluations. 


\section{Experiments}

Road crashes ontology was designed for experiments. It includes, among other things, cars and road signs hierarchies - both concepts inherit Existence class from base TBox. Actions include cars approaching, hitting, turn signalling and road line crossing, to name a few. A car can have colour, speed and licence plates. Four crash witness reports were created and then joined.

Report 1 (witness credibility - 0.3):

I was driving a red Opel Vectra. Its license plates are ABA2010. I approached Toyota Avensis. Its license plates are ABA255\%. I indicated left. I crossed the dashed line. I was driving $60 \mathrm{~km} / \mathrm{h}$. I hit Citroen C3. Its license plates are ABA3232. It was driving $100 \mathrm{~km} / \mathrm{h}$.

Report 2 (witness credibility - 0.6):

I was driving green Toyota Avensis. Opel Vectra approached me. It indicated left. Maybe it crossed the dashed line. It hit Citroen C3.

Report 3 (witness credibility - 0.9):

I was watching Opel Vectra with license plates ABA2010. No way it indicated left. It hit Citroen C3. Its license plates are ABA3232.

Report 4 (witness credibility - 0.3):

I was driving silver Citroen C3. Its license plates are ABA3232. I was driving 60 $\mathrm{km} / \mathrm{h}$. Red Opel Vectra crossed continuous line. Its license plates are ABA2010. I hit it.

The result of joining witnesses' reports is as following:

Red Opel Vectra with licence plates ABA2010 was riding $60 \mathrm{~km} / \mathrm{h}$. Silver Citroen C3 with licence plates ABA3232 was riding $80 \mathrm{~km} / \mathrm{h}$. The Opel Vectra maybe approached green Toyota Avensis with licence plates ABA255\%. It is very doubtful that the Opel Vectra turned on its signalization. It maybe overran a dashed line. It probably hit the Citroen C3.

It can be spotted that information coming from a witness with high credibility (report 3) has clearly higher final certainity factor (minimal certainity of Opel Vectra driver's turn signaling, greater certainity of Opel Vectra and Citroen C3 crash). Synonims among car instances were detected because of non-conflicting car identifiers (licence plates). The joined knowledge is a kind of all reports sum - contains even those facts that were not mentioned by all information sources, like colours of cars. The speed of Citroen C3 (numerical property), $80 \mathrm{~km} / \mathrm{h}$, was calculated as the mean of $100 \mathrm{~km} / \mathrm{h}$ and $60 \mathrm{~km} / \mathrm{h}$. It is worth noting that both values had the same certainity degrees in corresponding domain ABoxes, so the waged mean was not necessary here. Witness 2 credibility was high enough that even if he was not totally sure that Opel Vectra crossed the dashed line, 
it outclassed a certain sentence of low credible witness 4, who was saying the line was continous - there is a dashed line in the output ontology. Moreover, that version was confirmed by witness 1 .

It is worth noting that testing presented process of uncertain knowledge joining (grounding) is a complex task which cannot be truely automated. A proper domain ontology must be manually created, basing on provided abstract TBox, and several additional mappings, dependant on used domain, need to be provided as well - they were mentioned in the text. Every ontology is different in its structure, too, and that greatly influences the outcome of the used algorithms. It was assumed that automatically obtained results would be checked by confronting them with anticipated ones. It is subjective to each person reading them wether they are satisfying or not.

\section{Conclusions and Future Work}

The article presented the methodology of human-origined, uncertain knowledge grounding with the use of several ABoxes with uncertainities joining methods. Knowledge gaining was based on domian ABoxes with uncertainity values building. Each ABox was built from the text which contained domain knowledge of some particular information source. Several joining methods, like identifier-based joining, pattern-based joining, closure-based joining and action sequences joining, together with sentence certainity values evaluation, resulted in domain knowledge joining in such a way that maximized knowledge credibility and gave broader view on problem domain. Presented methodology supports different fact certainity degrees as well as credibilities of information sources. Possible fact conflicts and differently detailed event descriptions are also taken care of. The experiments from the previous section were to show effectiveness of used methods.

Future work will focus on knowledge gaining method extension. Inclusion of domain-aware agent [6] that will lead the dialogue with people, asking them proper questions, related to dialog context and history, is planned. That approach will eliminate some current disadvantages, like demanding knowledge about domain and system features from people.

Acknowledgements. We would like to thank Grzegorz Twarduś and Michał Pelczar for their help in writing this paper and system implementation.

\section{References}

1. Cognitive Science Laboratory, Princeton University: WordNet - a lexical database for the English language, http://wordnet.princeton.edu/

2. Euzenat, J., Le Bach, T., Barrasa, J., Bouquet, P., De Bo, J., Dieng, R., Ehrig, M., Hauswirth, M., Jarrar, M., Lara, R., Maynard, D., Napoli, A., Stamou, G., Stuckenschmidt, H., Shvaiko, P., Tessaris, S., Van Acker, S., Zaihrayeu, I.: State of the art on ontology alignment. Knowledge Web Deliverable, Technical Report, INRIA (2004) 
3. Fridman, N., Musen, M.: SMART: Automated Support for Ontology Merging and Alignment. In: Twelth Workshop on Knowledge Acquisition, Modeling and Management, Banff, Canada (1999)

4. Gruber, T.: What is an Ontology, http://www-ksl.stanford.edu/kst/what-is-an-ontology.html

5. Haase, P., Motik, B.: A Mapping System for the Integration of OWL-DL Ontologies. In: IHIS 2005, Bremen (November 2005)

6. Josyula, D.P., Fults, S., Anderson, M.L., Wilson, S.: Application of MCL in a dialog agent

7. Kalfoglou, Y., Schorlemmer, M.: Ontology Mapping: The State of the Art. The Knowledge Engineering Review 18(1), 1-31 (2003)

8. Łuszpaj, A., Szymański, K., Zygmunt, A., Koźlak, J.: The Process of Integrating Ontologies for Knowledge Base Systems. In: 7th Software Engineering Conference, Cracow (2005)

9. Niles, I., Pease, A.: Towards a Standard Upper Ontology. In: Proceedings of the 2nd International Conference on Formal Ontology in Information Systems. FOIS-2001 (2001)

10. Pan, J.Z., Stamou, G., Tzouvaras, V., Horrocks, I.: f-SWRL: A Fuzzy Extension of SWRL

11. Pazienza, M.T., Stellato, A., et al.: Ontology Mapping to support ontology-based question answering. In: 4th International Semantic Web Conference (ISWC-2005), Galway, Ireland (November 2005)

12. Pinto, H.S., Martins, J. P.: Some Issues on Ontology Integration. Portugal (2001)

13. Stoilos, G., Stamou, G., Tzouvaras, V., Pan, J.Z., Horrocks, I.: Fuzzy OWL: Uncertainty and the Semantic Web

14. Straccia U.: Answering Vague Queries in Fuzzy DL-Lite 